\section{Advantages of $360^{\circ}$ Virtual Forest Tours to Supplement Academic Forestry Education}

GI_Forum 2021, Issue 2

Page: 34 - 44

Full Paper

Corresponding Author: t.foehrder@twistedminds.com.de DOI: 10.1553/giscience2021_02_s34

\author{
Torben Foehrder, Jan-Peter Mund and Peter Spathelf \\ Eberswalde University for Sustainable Development, Germany
}

\begin{abstract}
$360^{\circ}$ virtual forest tours (VFTs), created by state-of-the-art virtual reality technology, contribute to the progression of academic e-learning and forest management. The article proposes a conceptual framework for $360^{\circ} \mathrm{VFT}$ production and use to supplement field courses. Reflective insights into the authors' own learning process in the production of $360^{\circ}$ VFTs, and a consideration of the general challenges of the medium are also presented. To conclude, the article summarizes the advantages of $360^{\circ} \mathrm{VFT}$ for forestry education in an academic context.
\end{abstract}

\title{
Keywords:
}

virtual forest tours, forestry education, virtual reality, e-learning

\section{Introduction}

Excursions, field exercises and real-world laboratories are core elements of practice-oriented university didactics in education for sustainable development (ESD). The context-sensitive learning objectives of ESD cover knowledge transfer and cognitive experience, especially in the areas of nature conservation, ecology and forestry. Immersive and interactive learning is a modern didactic method in which 3D animation technology can be used to immerse learners in virtual realities (VR) and enable them to interact with so-called digital twins. This method is already widely used in technical sciences. It has been proven to promote learning efficiency by allowing learners to revisit the VR experience multiple times, in combination with more traditional forms of learning - or to use Pantelidis's terminology, through the combination of symbolic and experiential information (Pantelidis, 2009). The technological advancements in augmented, mixed and virtual reality (AR/MR/VR) applications support learning in cases of the physical or ethical inaccessibility of the object of study. Interactive 3D immersive learning spaces' bridge barriers of space and time, and enable focusing via AR/MR/VR on parameters or ratios in ecosystems that are invisible or not immediately comparable (e.g. size of tree stems compared to crown height; or crown size in relation to leaf cover, which can only be determined in different seasons). In the light of ongoing developments by the GIS Laboratory of the University for Sustainable Development Eberswalde, this article describes the creation 
of a practical $360^{\circ}$ virtual forest tour $\left(360^{\circ} \mathrm{VFT}\right)$, which can be used as a tool for teaching and project coordination in forestry education. With suitable AR solutions already available (Mund \& Müller, 2019), the development of VR teaching methods is the next logical step. The scope of the article includes the practical integration of the technology for individuals and institutions interested in the possibilities that $360^{\circ}$ VFTs have to offer. An analysis of the didactical methods and advantages of the medium is not part of this article, as this would considerably extend the cover of the research. For an examination of immersive VR technology and learning theories for implementation in higher education contexts, see e.g. Radianti et al. (2020).

$360^{\circ}$ VFTs have become popular in environmental education as a way of informing people about how forestry benefits humans and wildlife (Kershaw, 2020). Transferring the didactic tool into an academic context serves as a supplement for field trips and partially solves limitations on financial and logistical resources (Reque Kilchenmann, García Ochoa, \& Spathelf, 2017). To identify the contributions of $360^{\circ}$ VFTs to higher education in forestry, the authors examined their personal experiences of $360^{\circ} \mathrm{VFT}$ production and the technical long-term implementation of the tours. A basic framework for the creation and comparison of $360^{\circ}$ VFTs is presented, and the advantages of the medium are critically assessed in relation to the efforts required for successfully producing VFTs.

\section{Materials and methods}

Investigation into $360^{\circ}$ VFTs as a possible tool for academic research and teaching was carried out using a basic technical setup. The equipment consisted of various digital single-lens reflex (DSLR) cameras, positioned in a panoramic head (Manfrotto, Cassola, Italy), mounted on a standard tripod in the field, and operated manually. Execution was constantly refined to evaluate the quickest and most reliable routine for photographing spots of interest for later digitalization. The image data acquired were then processed in PTGui (a software package that is currently available) and Panotour Pro (which is no longer released). This allowed the twodimensional pictures to be re-arranged in a virtual simulation of the precise locations photographed.

Using PTGui, the photographs taken in the field were merged to create a spherical simulation of the site (see Figure 1). The software includes an additional masking tool tool for favouring or excluding areas in individual source images, as is found in most types of image-editing software. Basic functions such as the manual adjustment of anchor points shared by images and their placement within the virtual sphere, as well as simple graphic-adjustment tools, are also part of PTGui. The files produced allow a $360^{\circ}$ experience of the virtual replica of a realworld location. To connect these files to an interactive tour, implement additional digital content, and generate a publishable HTML file, the Panotour Pro software by Kolor (Figure 2) proved sufficient, but distribution of the product was suspended and technical support discontinued during the period when this study was being carried out. 

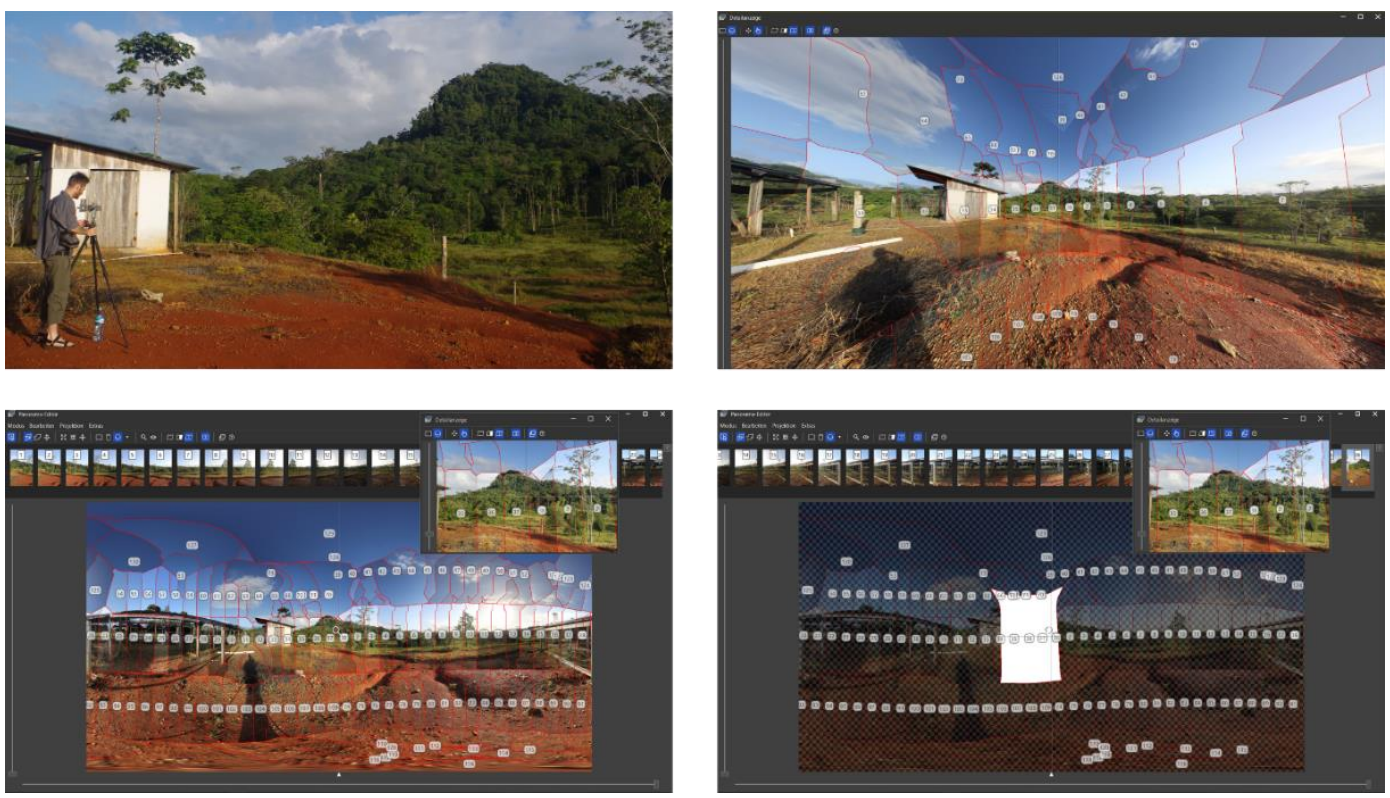

Figure 1: Transformation and distortion of single photographs taken by DSLR cameras in the creation of a spherical virtual replica using PTGui. From top left to bottom right: generation of photographic source files; merged images in spherical simulation; mosaic display of source files; distortion of source files within the panorama

The combined efforts of several projects allowed the production of various $360^{\circ} \mathrm{VFT}$ s for the purposes of academic knowledge transfer and project transparency. In this way, methods of large-scale forestry management could be visualized and compared. The aspects looked at included areas for practical fieldwork in forestry (marteloscopes), international ecosystem comparison, reforestation areas, and areas of storm damage and their structural development over time. Various ways of making the tours available for user interaction were compared and tested for accessibility, ease of application and sustainable data management. 

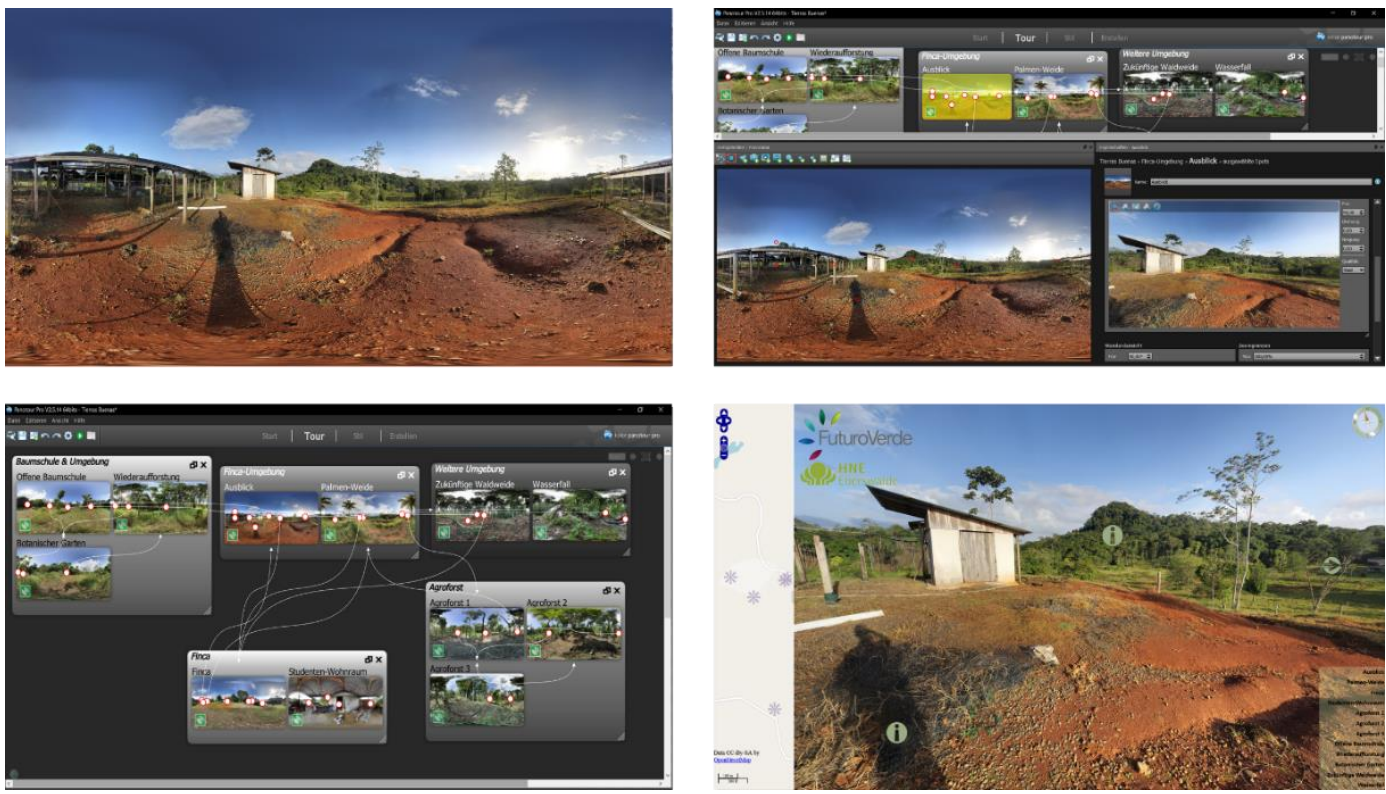

Figure 2: Implementation of the previously generated panoramic image into a virtual tour, including interactive and connective elements, using Panotour Pro. From top left to bottom right: panoramic image generated; implementation of spatial references and information; combining scenes into a tour; final tour as viewed in a web browser.

\subsection{Subsequent reflexion}

Based on their experiences in the projects undertaken, the authors refined the conceptual framework for the content-related production of a $360^{\circ}$ VFT. After understanding the technological conditions needed to produce photographic material which meets the requirements for digital progression, the authors next examined the viewer's experience of the tour. For academic e-learning concepts in relation to forestry, tours that are publicly available were compared to the project results in order to extract preferred features. Additionally, a review of scientific and popular literature connected to forest-related VR research was carried out with a view to identifying possible implementations of $360^{\circ}$ VFTs and their academic value.

\section{$3 \quad 360^{\circ} \mathrm{VFT}$ production}

The production of a $360^{\circ}$ VFT requires the same conceptual framework as that found in most projects focusing on public interaction and media presentation. Informational content, contextual storyboard, publishing environment and technological capacities are defining factors for the composition of the tour. The extent of the information and additional data to include can then be determined. Interested parties may consider outsourcing the creation of a $360^{\circ} \mathrm{VFT}$ as a way of reducing time, effort and costs while still achieving satisfactory results; indeed, there are more and more professional service providers creating virtual tours. The 
most prominent companies in this domain are Google Tour Creator, RICOH360 and 3DVista. To create or modify a $360^{\circ}$ VFT, using a third party or in-house resources, a sound understanding of the basic procedures may shorten the development process and aid in the design of a working concept.

For the creation of a $360^{\circ} \mathrm{VFT}$, interested parties first need to generate suitable visual content for the spherical replication - i.e. panoramic photographic images. This is followed by the implementation of additional information, choosing a didactic connection between scenes, and embedding the tour in its publishing environment. The continuous development of equipment and software makes the technical procedures in $360^{\circ}$ VFT production increasingly easy, but creative decisions for the integration of content require a further skill set in addition to subjectknowledge of forestry. Depending on the target group and the purpose of a $360^{\circ} \mathrm{VFT}$, creators may consider deepening their understanding of digital and visual media, immersive didactics and interdisciplinary knowledge transfer.

\subsection{Preparatory framework}

Most important during the outlining phase of a $360^{\circ} \mathrm{VFT}$ is determining how much time will have to be invested for individual stages of the project. Furthermore, it must be kept in mind that acquiring appropriate image data requires favourable weather conditions. To avoid accumulating unnecessary data, the number and location of scenes to be shot should be established before starting the fieldwork. The choice of equipment and software (see Section 3.2) determines how the tour will be published and what modifications will be possible, and should therefore be addressed as early as possible.

\subsection{Technical requirements}

Integrated functions for the generation of panoramic image files can be found in conventional hand-held photographic devices (mobile phones, compact cameras, or point-and-shoot devices like GoPro models). Unmanned aerial vehicles (UAVs) are the most convenient way to inspect hard-to-reach locations while assuring exact geographical references. Higher-priced models commonly include settings for panorama shots (Muliawan, 2017). For high-resolution image data and the option to adjust settings according to the circumstances in the field, manually operated DSLR cameras are sufficient. However, full-panoramic $\left(360^{\circ}\right)$ footage can only be produced by specific state-of-the-art cameras. These are not currently available commercially as integrated components of UAVs (Feist, 2020).

Digital processing of the photographs to create spherical virtual environments requires less computing capacity than, for example, editing movie clips, but extracting clipped scenes from high-resolution image data is still very time-consuming. To guarantee fluid progress on the technical work involved and editing procedures, devices rely mainly on graphic components, such as high GPU capacities, and adequate virtual working storage. The accumulation of data can cause files for a single VFT to quickly exceed 10GB. Adequate hard-drive space therefore needs to be ensured. 


\subsection{Project stages of VFT production}

These basic guidelines offer the essential procedures for producing a $360^{\circ}$ VFT. The concept can be applied to any location and desired subject.

\section{Draft of the project's scope}

A preliminary definition of the $360^{\circ}$ VFT subject is reached by consolidating the knowledge that is to be transferred and identifying the geographical references to be included. Also during the planning phase, the means of transport to the locations for photographic fieldwork, the creative partners in the project and their intentions for specific content, and the choice of equipment and publication platform need to be decided. The conceptual framework determining how many scenes to create and their sequence also need to be decided in relation to the target viewers of the $360^{\circ}$ VFT.

\section{Fieldwork and post-processing}

A basic understanding of photography is necessary to achieve suitable photographic image data at the geographic location of interest. Differing weather and light conditions require constant adaptation for optimal source material. The effort invested may vary widely based on the equipment used and the desired level of detail.

The individual photographic image files are merged into a single larger image file, which can be displayed using specific software as a virtual $360^{\circ}$ spatial experience of the scene. Given appropriate source material and software, creating a spherical combination of the individual files requires minimal manual graphical corrections or selection/omission of individual source files in the mosaic display (Figure 1).

\section{Tour creation and publication}

Subsequently, the individual scenes are connected to form a virtual tour. If augmented by geographical references, the sequence of the individual scenes can deliver an understanding of the environmental conditions at the geolocation, at a specific point in time (Figure 2). Metadata or the appropriate EXIF files should therefore always be accessible for auxiliary information - but via icons or other interactive functions, so as not to obstruct the viewer's undisturbed field of vision. Trade-offs between fostering the benefits of an intuitive understanding of the virtual replicated environment, ease of access to supplementary technological components, and software limitations are to be expected during this phase.

When a $360^{\circ}$ VFT is created for public access, online lessons or as a stand-alone production, webhosting, remote access or a server structure is necessary to deliver the tour to interested viewers. Hosting services like WordPress or Wix have published technical expansions to integrate virtual $360^{\circ}$ content. Software that can produce virtual tours generates usable data formats (HTML5 or JavaScript are common) and may include complementary software to present the created tour via an Intranet or a local host. If technical training and resources are available, the tours may also be made available through in-house webhosting. 


\section{Utilization of $360^{\circ}$ VFT}

Especially in the context of a worldwide pandemic, the advantages of remote-access study courses and virtual excursions have become ever more apparent. A virtual replication of realworld forest stands allows for repeatable experience in a specific area of interest, independently of the current weather conditions, season of the year or transport. The exchange of (detailed, computerized) academic knowledge in connection to forestry (e.g. types of vegetation and structural composition, growth over time, or effects of natural disasters) can be fostered by the opportunities offered by $360^{\circ} \mathrm{VFT}$, and vice versa.

One example of $360^{\circ}$ VFTs being used to supplement or complement traditional courses of study, classroom teaching methods and fieldwork are the teaching units designed by the Institute for Sustainable Forest Management Research, University of Valladolid, Spain (Figure 3), which are based on interactive online walks through real forest stands (Instituto Universitario de Investigación Gestión Forestal Sostensible (iuFOR), 2018). Documentation of experiments, of extraordinary events such wildfires or storms, of harvesting, planting or fencing allows for immediate visual comparison of plant growth, for example, and offers a holistic perspective on the ecosystem (Figure 4). The European-Vietnamese Higher Education Network for Sustainable Forest- and Bio-Economy, another instance of a $360^{\circ}$ VFT used in education, aims to examine issues in a multidisciplinary fashion and to compare different forest ecosystems on a global scale.

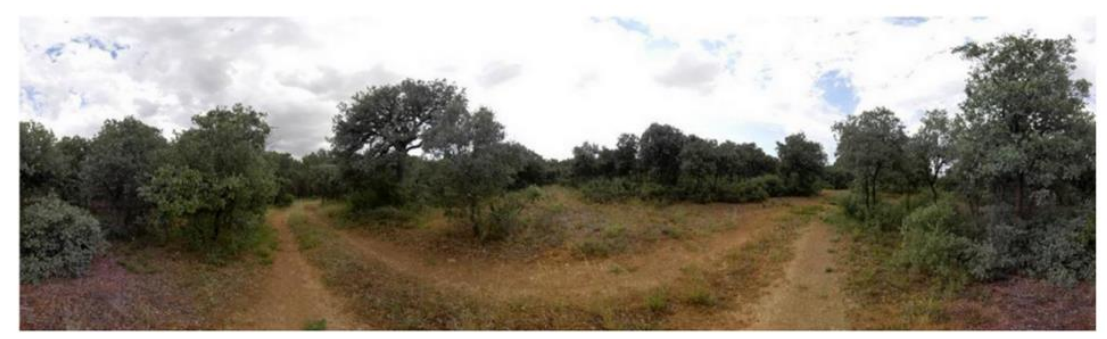

Didactic materials

Virtual Visits

\section{MARTELOSCOPE XAMK_South-Eastern Finland University of Aplied \\ Sciences (Mikkeli)}

Marteloscope XAMK (Mikkeli, Finland)

Key words: Forestry, silviculture, harvesting, thinning, clear-cut, tending, regeneration, Hippala, Mikkeli, BIOECON.EU, ERASMUS MUNDUS

HOME

Fullscreen : http://sostenible.palencia.uva.es/rutas/Marteloscope_XAMK/index.html

View Virtual Visit $>$

\section{MARTELOSCOPE Thai Nguyen University of Forestry_Vietnam (Virtual Forest Tour)}

MARTELOSCOPE TUAF Thai Nguyen University of Forestry, Vietnam

Figure 3: Screen shot of $360^{\circ}$ VFT to supplement academic field courses sostenible.palencia.uva.es (Instituto Universitario de Investigación Gestión Forestal Sostensible (iuFOR), 2018) 

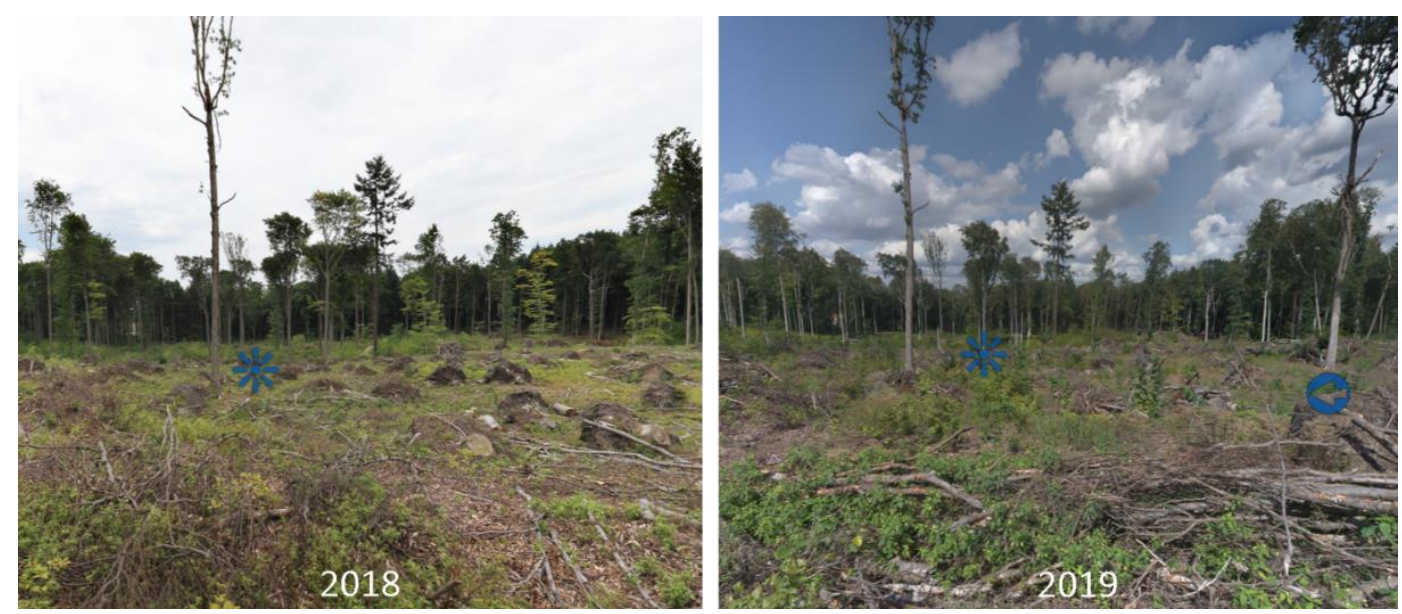

Figure 4: Sample images showing different site conditions after storm damage (Mund, Beiler, \& Manh, BioEcoN, 2019)

The development of a virtual teaching environment for owners of small-scale forests to ensure adaptation of their stands to future conditions caused by climate change is the subject of a recent project by the Department of Information Management and Information Systems at the University of Osnabrück (Thomas, 2021). This example of the use of $360^{\circ} \mathrm{VFT}$ in higher education is aimed at young forest owners who have little forest management experience, for the identification of tree species, assessment of forest stands, identification of habitats, timber harvesting, and rejuvenation.

\section{Discussion}

Setting up a suitable collection of $360^{\circ}$ VFTs for academic e-learning and knowledge transfer requires a considerable investment of time, suitable equipment, relevant media competence, photography and programming skills, and secure web hosting. Consequently, virtual tour production and webhosting are now carried out predominantly by businesses instead of individual creators. Cancellation of support for the Panotour Pro production software by developer Kolor was a further complexity encountered during the learning process.

The importance of ethics and moral sensitivity to be integrated into new technologies reached new levels with the development of VR. Referred to as 'anticipatory technology ethics' by Philip A. E. Brey (Brey, 2012) and 'responsible research and innovation' by Hilary Sutcliffe (Sutcliffe, 2011), the responsibilities and moral obligations of technology designers to the public may include a wider long-term view, taking into account social involvement, environmental impacts and other repercussions. Predictions, forecasting impacts, and evaluating and elaborating on possible consequences need to be integrated into the development of VR applications to guarantee early identification of issues with openness and transparency. Additionally, profound acknowledgement of active, real, lived experience needs to be a fundamental psychological element in VR development for the provision of a 'positive' 
experience in a virtual environment, which is supposed to stay as close to reality as possible (Kenwright, 2019).

\subsection{Advantages of $360^{\circ}$ VFT in forestry education}

The potential of $360^{\circ}$ VFTs to offer visual impressions of environmental conditions at a particular geographical location that can be compared with each other makes this a holistic and comprehensive medium for understanding the complex interdependencies that exist within a forest ecosystem. The level of immersion provided by $360^{\circ} \mathrm{VFT}$ s cannot be accomplished by traditional textual or photographic learning materials. By connecting geographical references, extracted in semi-autonomous fashion from existing image metadata, and photo-optic panoramic portrayals of a location, remote users are able to immerse themselves in the scene and to experience its content in an interdisciplinary and unbiased way. Further implemented data, accessed via intuitive and meaningfully (spatially) oriented links/icons within the scenes, can supplement a tour. In this way, the visual concept of a $360^{\circ} \mathrm{VFT}$ supports memorizing, understanding, and the creation of links between different pieces of information (Kouyoumdjian, 2012). VR implementations, due to their intuitive, digital and transdisciplinary nature, facilitate increased learning engagement, higher accuracy in planning processes, decreased costs of prototype development, as well as the generation of new ways of communicating and interacting between collections of data, products, locations and stakeholders (Marr, 2017).

Regarding forestry education specifically, the digital recreation of real-world forest structures in a $360^{\circ}$ VFT provides an objective picture to unify all available data, combined with analytical tools to support collaborative decision-making and stakeholder engagement. Cost reduction and increased productivity are possible by minimizing the reliance on field trips and using $360^{\circ} \mathrm{VFT}$ for forest management decisions (Roeser, 2020). Current $360^{\circ} \mathrm{VFT}$ applications include updating forest asset information, automatization of data integration via remote sensing, and improvement of forest machinery and management simulators for staff training (Fabrika, Valent, \& Mokroš, 2016). Highly immersive VR simulations are of increasing value for school purposes due to their independence of time and location. They also provide a riskfree environment and room for mistake-driven experiences (Pappas, 2017). In the long term, ambitions are set to create digital replicas of forests, including the modelling of every tree in terms of its location, height, diameter and species. It will become possible to visit timber transactions virtually and compare management decisions. The simulation of stand conditions after the application of different management decisions will be another convenience of $360^{\circ}$ VFTs (Hofmann \& Jumppanen, 2017).

\section{Prospects and developments}

Digital replications of real-world forest stands are expected to become further enhanced. They will provide geospatial references for silvicultural objects improved by the integration of multisensory data, generated by autonomous and manually-operated vehicles in forest operations. Using photogrammetric or LiDAR technology in $360^{\circ} \mathrm{VFT}$ could allow the display of point cloud data in a graphically enhanced way within a tour. Viewers would be able to carry out 
assessment tasks for which natural human perceptual skills are required, without the restrictions of a conventional display screen (Chinthammit, 2017). Calculations performed by integrated functions within the software could reduce manual documentation and automate basic assessments (Fabrika, Valent, \& Mokroš, 2016). It may soon be possible to create autonomous inventories, as shown by developments discussed in (Mohan, et al., 2017). Given the increasing accuracy of sensor technologies, inventories will achieve greater levels of reliability through autonomous, technological means than human observation alone is able to accomplish.

\section{References}

Bardi, J. (2020, 09 21). Marxent. (Marxent, Editor) Retrieved 01 31, 2021, from What is Virtual Reality? VR Definition and Examples: https://www.marxentlabs.com/what-is-virtual-reality/

Brey, P. A. (2012, 04 04). Anticipatory ethics for emerging technologies. NanoEthics, 6(1-13). doi:https://doi.org/10.1007/s11569-012-0141-7

Chinthammit, D. W. (2017). ForestTECH. (I. Ltd., Interviewer) Retrieved 01 31, 2021, from Virtual reality and it's use in local forests: https:/ / fridayoffcuts.com/dsp_article.cfm?id=745\&aid=8903

Fabrika, M., Valent, P., \& Mokroš, M. (2016). Sektion Ertragskunde im Verband Deutscher Forstlicher Versuchs- und Forschungsanstalten. (T. U. Faculty of Forestry, Ed.) Retrieved 01 31, 2021, from Forest Landscape Simulations in Immersive Virtual Reality: http://sektionertragskunde.fvabw.de/2016/08_Fabrika_et_al.pdf

Feist, J. (2020, 07 27). Drone Rush. Retrieved 01 31, 2021, from Best drones with 360 degree camera: https://dronerush.com/best-drones-360-degree-camera-10725/

Google Street View. (2021). Google Street View. (Google Street View) Retrieved 01 31, 2021, from Take a look at all the Street View mapping stories: https://www.google.com/intl/en$\mathrm{GB} /$ streetview/case-studies/

GoPro, Inc. (2020). Kolor Panotour. (GoPro, Inc.) Retrieved September 16th, 2020, from Kolor: https://www.kolor.com/

Hofmann, C., \& Jumppanen, J. (2017). Metsä Group - Forerunner in sustainable bioeconomy. (M. Group, Editor) Retrieved 01 31, 2021, from Virtual forests are coming: https://www.metsagroup.com/en/Campaigns/IntelligentMetsa/intelligentforest/Virtual-forestsare-coming/Pages/default.aspx

Instituto Universitario de Investigación Gestión Forestal Sostensible (iuFOR). (2018, 01 11). INICIO | Gestión Forestal Sostensible. Retrieved 01 31, 2021, from Virtual Forest Tours: http:/ / sostenible.palencia.uva.es/content/virtual-forest-tours

Kenwright, B. (2019, 01 14). Technology and Society. Retrieved 01 31, 2021, from Virtual Reality: Ethical Challenges and Dangers: https://technologyandsociety.org/virtual-reality-ethicalchallenges-and-dangers/

Kershaw, A. (2020, 01 14). The Ecologist. (T. Ecologist, Editor) Retrieved 01 31, 2021, from Virtual forest tours for children: https://theecologist.org/2020/jan/14/virtual-forest-tours-children

Kouyoumdjian, H. (2012, 07 20). Psychology Today. Retrieved 01 31, 2021, from Learning Through Visuals: https://www.psychologytoday.com/us/blog/get-psyched/201207/learning-throughvisuals

Marr, B. (2017, 07 31). Forbes. (Forbes, Editor) Retrieved 01 31, 2021, from The Amazing Ways Companies Use Virtual Reality For Business Success:

https://www.forbes.com/sites/bernardmarr/2017/07/31/the-amazing-ways-companies-usevirtual-reality-for-business-success/ 
Mohan, M., Silva, C. A., Klauberg, C., Jat, P., Catts, G., Cardil, A., . . Dia, M. (2017, 09 11). Individual Tree Detection from Unmanned Aerial Vehicle (UAV) Derived Canopy Height Model in an Open Canopy Mixed Conifer Forest. Forests, 8(9). doi:https://doi.org/10.3390/f8090340

Muliawan, R. C. (2017, 05 08). SkyPixel. Retrieved 01 31, 2021, from Tips for 360-Degree Aerial Photography: https://store.dji.com/guides/tips-360-degree-aerial-photography/

Mund, J.-P., Beiler, K., \& Manh, V. V. (2019). BioEcoN. Retrieved 07 15, 2021, from Virtual Forest Tours: http://bioecon.eu/virtual-forest-tours/

Mund, J.-P., \& Müller, S. (2019). Augmented Reality and Mobile GIS as Tools for Teaching Datacollection in the Context of Forest Inventories. (U. f. Eberswalde, Ed.) GI_Forum, pp. 129-143. doi:10.1553/giscience2019_02_s129

New House Internet Services B.V. (2020). PTGui. (New House Internet Services B.V.) Retrieved September 16th, 2020, from PTGui: https://www.ptgui.com/

Pantelidis, V. S. (2009). Reasons to Use Virtual Reality in Education and Training Courses and a Model to Determine When to Use Virtual Reality. (C. o. Department of Library Science, Ed.) THEMES IN SCIENCE AND TECHNOLOGY EDUCATION, 2(Special Issue), pp. 59-70. Retrieved 07 15, 2021, from https:/ / files.eric.ed.gov/fulltext/EJ1131313.pdf

Pappas, C. (2017, 02 11). eLearning Industry. Retrieved 01 31, 2021, from 6 Tips To Use Virtual Reality In Online Training: https://elearningindustry.com/tips-use-virtual-reality-online-training

Radianti, J., Majchrzak, T. A., Fromm, J., \& Wohlgenannt, I. (2020, April). A systematic review of immersive virtual reality applications for higher education: Design elements, lessons learned, and research agenda. Computers \& Education, 147. doi:https://doi.org/10.1016/j.compedu.2019.103778

Reque Kilchenmann, J., García Ochoa, M., \& Spathelf, P. (2017). Bringing the forest to the classroom: developing virtual tours in forestry. In 1. I. (ICEE21C) (Ed.), New competences in Engineering Education in the area of sustainability and university social responsibility (pp. 23-26). Castelló de la Plana: Universitat Jaume I.

Roeser, D. (2020, 04 17). The University of British Columbia. Retrieved from How Virtual Reality Can Aid Land-Based Resource Management and Operation Planning: https:/ / forestry.ubc.ca/research/how-virtual-reality-can-aid-forest-operations/

Sutcliffe, H. (2011, 05 16-17). European Commission. Retrieved from Responsible Research and Innovation Report: https://ec.europa.eu/programmes/horizon2020/sites/default/files/rrireport-hilary-sutcliffe_en.pdf

Thomas, O. (2021, 05 01). Universität Osnabrück. Retrieved 007 15, 2021, from Department of Information Management and Information Systems (IMWI): https://www.wiwi.uniosnabrueck.de/fachgebiete_und_institute/informationsmanagement_und_wirtschaftsinformatik_ prof_thomas/projekte/virtual_reality_forestry_training.html

Vitec Imaging Solutions Spa. (2021). Manfrotto. Retrieved 07 15, 2021, from Virtual reality panoramic head w/ multiple sliding plates: https://www.manfrotto.com/global/virtual-reality-panoramichead-w-multiple-sliding-plates-mhpanovr/ 